\title{
Effect of Hydrogenation on the Electronic Structure of HoNiSn - Ab Initio Calculations
}

\author{
A. JEZIERSKI ${ }^{a}$, A. SZAJEK ${ }^{a}$ AND M. JURCZYK ${ }^{b}$ \\ ${ }^{a}$ Institute of Molecular Physics, Polish Academy of Sciences, M. Smoluchowskiego 17, 60-179 Poznań, Poland \\ ${ }^{b}$ Institute of Material Science and Engineering, Poznań University of Technology \\ pl. M. Skłodowskiej-Curie 5, 60-965 Poznań, Poland
}

\begin{abstract}
The electronic and magnetic properties of HoNiSn alloy are studied by ab initio full relativistic full potential local orbital method within the local density approximation. The electronic structures are reported for two crystallographic structures: orthorhombic TiNiSi type and the hexagonal ZrNiAl-type structure. We also study the influence of hydrogenation on the electronic structure of $\mathrm{HoNiSnH}_{0.66}$. Ab initio calculations have shown that in the both type of structures the Fermi level is located at the $4 f$ peak of Ho, however we observed the modification of $3 d$ peaks from nickel atoms below the Fermi energy. The hydrogenation leads to increase of the density of states at the Fermi level.
\end{abstract}

PACS numbers: 71.20.-b, 71.15.Ap, 71.15.Mb, 71.20.Eh, 71.20.Lp

\section{Introduction}

The experimental study of HoNiSnD alloys [1-4] have shown that deuterium absorption leads to contraction of volume of $0.64 \%$. Such contraction was associated with structural phase transition from orthorhombic TiNiSi-type structure (space group Pnma, No. 62) (Fig. 1) to the hexagonal $\mathrm{ZrNiAl}$-type structure (space group $P 62 \mathrm{~m}$, No. 189) (Fig. 2). The effect of hydrogenation of HoNiSn was studied experimentally recently by Yartys et al. [1], Szytula et al. [2] and Yakinthos et al. [3]. The volume contraction is connected to the instability of the TiNiSi structure with respect to insertion of interstitial atoms and leads to transformation to the ZrNiAl-type structure.

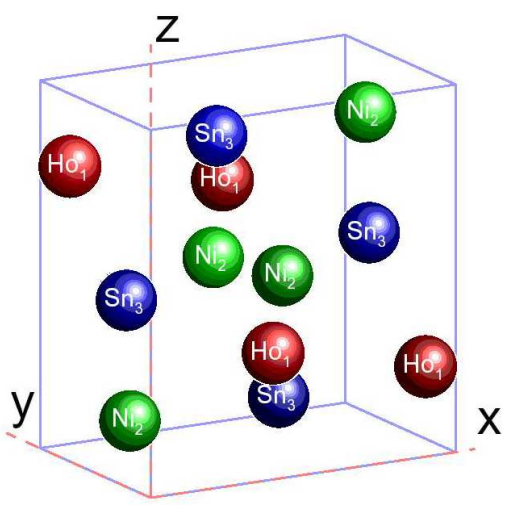

Fig. 1. Crystal structure of orthorhombic HoNiSn (space group No. 62).

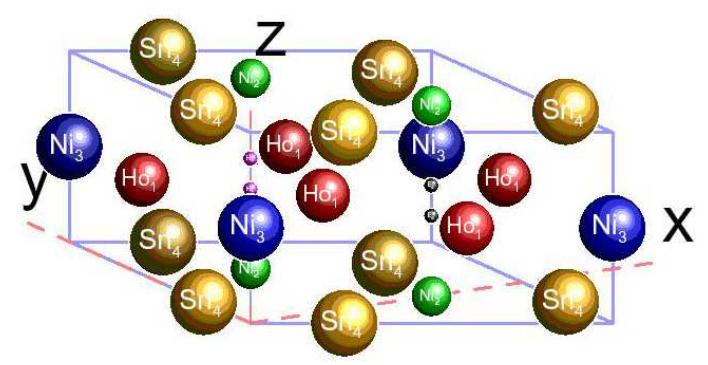

Fig. 2. Crystal structure of hexagonal $\operatorname{HoNiSnH(Vc)~}$ (space group No. 189).

The neutron diffraction measurements [2] have shown that deuterium atoms occupied the tetrahedral $4 \mathrm{~h} \mathrm{Ho}_{3} \mathrm{Ni}$ sites. The Ho magnetic moment in HoNiSn was estimated as $8.27 \mu_{\mathrm{B}}$, however in $\mathrm{HoNiSnD}_{0.67}$ its value was reduced to $5.36 \mu_{\mathrm{B}}$.

This interesting future motivated us to study the electronic and magnetic properties of HoNiSn in orthorhombic and hexagonal type structures. We also examined the influence of hydrogen on the electronic structure near the Fermi energy. The outline of method of calculation is given in Sect. 2. The results and conclusions are presented in Sects. 3 and 4, respectively.

\section{Method of calculations}

The electronic and magnetic properties of ordered HoNiSn were calculated by using the full relativistic full potential local orbital (FPLO) method within the local density approximation (LDA) [5]. The electronic 
structure of $\mathrm{HoNiSnH}_{0.66}$ alloy was performed by full-potential scalar relativistic local orbital method within the coherent potential approximation (FPLO-CPA) [6]. In both methods the exchange correlation potential was used in the form of Perdew and Wang [7]. In the band calculations we assumed the following configurations of atoms: Ho: core + valence electrons $(4 d 4 f 5 s 5 p 6 s 6 p 5 d)$; $\mathrm{Ni}$ : core + semicore $(3 s 3 p)+$ valence electrons $(4 s 4 p 3 d)$; Sn: core + valence electrons $(4 s 4 p 5 s 5 p 4 d)$. The self-consistent calculations were carried out on a $k$ mesh of $847 k$-points in each direction of the irreducible Brillouin zone in the case of $\mathrm{HoNiSnH}_{0.66}$ and $1331 k$-points for the ordered HoNiSn alloys. The self-consistent criterion was equal to $10^{-8}$ Ry for the total energy. The calculations were performed for the experimental values of the lattice parameters (Table I) [1, 2]. The HoNiSn crystallizes into orthorhombic TiNiSn-type structure (Fig. 1) and after hydrogenation the crystal structure goes into hexagonal ZrNiAl-type structure (Fig. 2). The positions of atoms in both unit cells are listed in Tables II and III. In order to study the effect of change of crystal structure on the electronic and magnetic properties of HoNiSn alloys, we have performed the self-consistent $a b$ initio calculations for both types of crystal structures. In the case of the hexagonal ZrNiAl-type structure we put the vacancies into the $4 \mathrm{~h}$ position.

TABLE I

The experimental values of lattice parameter of HoNiSn alloys [1, 2].

\begin{tabular}{c|c|c|c}
\hline \hline Space group & $a$ & $b$ & $c$ \\
\hline Pnma (62) [1] & 7.0682 & 4.4396 & 7.643 \\
P62m (189) [2] & 7.2402 & 7.2402 & 3.936
\end{tabular}

TABLE II

Crystal structure data for HoNiSn (space group No. 62) [2].

\begin{tabular}{c|c|c|c}
\hline \hline Atom & $x$ & $y$ & $z$ \\
\hline $\mathrm{Ho}$ & 0.4924 & 0.25 & 0.2992 \\
$\mathrm{Ni}$ & 0.2955 & 0.25 & 0.5834 \\
$\mathrm{Sn}$ & 0.8079 & 0.25 & 0.5871
\end{tabular}

TABLE III

Crystal structure data for $\mathrm{HoNiSnH}(\mathrm{Vc})$ (space group No. 189) [1].

\begin{tabular}{c|c|c|c}
\hline \hline Atom & $x$ & $y$ & $z$ \\
\hline Ho 3g & 0.5984 & 0.0 & 0.5 \\
Ni 2c & $1 / 3$ & $2 / 3$ & 0.0 \\
Ni 1b & 0 & 0 & 0.5 \\
Sn 3f & 0.259 & 0 & 0 \\
Vc (H) 4h & $1 / 3$ & $2 / 3$ & 0.58
\end{tabular}

\section{Results and discussion}

The electronic and magnetic properties of HoNiSn alloys were performed for non-magnetic and the spin-polarized systems. In Figs. 3 and 4 we presented the total density of states and the contributions from Ho, $\mathrm{Ni}$ and $\mathrm{Sn}$ atoms for non-magnetic HoNiSn alloy (space group No. 62). Figure 3 gives the results for scalar-

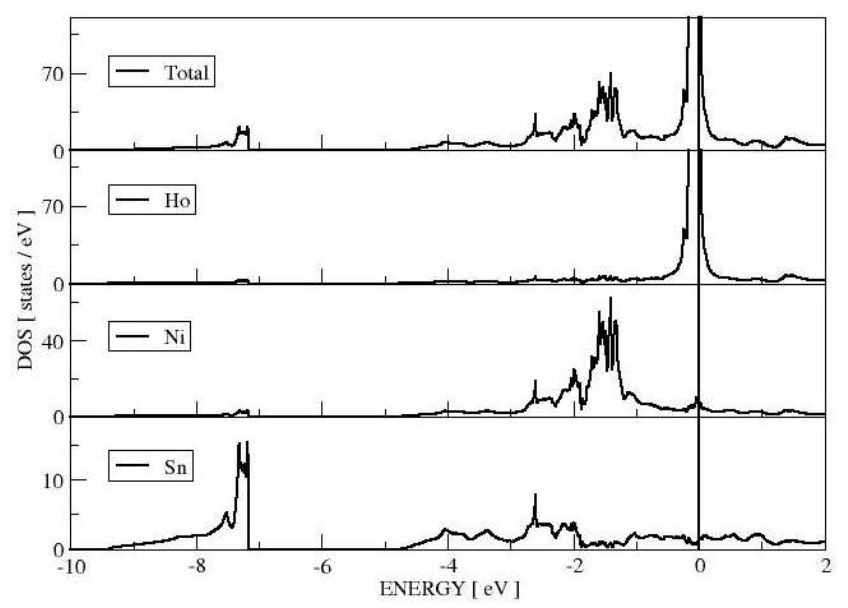

Fig. 3. Total and partial electronic density of states of HoNiSn (scalar-relativistic, space group No. 62).

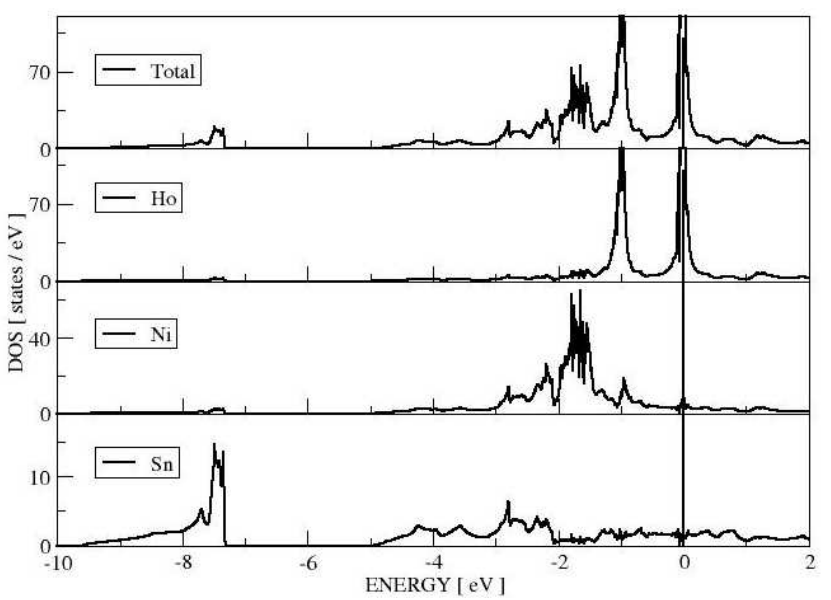

Fig. 4. Total and partial electronic density of states of HoNiSn (full-relativistic, space group No. 62).

-relativistic and Fig. 4 for full-relativistic including spinorbit coupling method. In both figures the main contribution near the Fermi energy comes from $4 f$ states of Ho atoms. The broad peaks in the region of energy $-4 \mathrm{eV}<E<-1 \mathrm{eV}$ give the contributions from nickel atoms. The $5 s$ states of $\mathrm{Sn}$ are visible below $-7 \mathrm{eV}$. The main difference between plots in Figs. 3 and 4 is the splitting of $4 f$ states of holmium. The density of states at the Fermi energy $N\left(E_{\mathrm{F}}\right)$ is 201.93 and 155.83 (states/ (eV f.u.)) for scalar-relativistic and full-relativistic calculations, respectively. 
The results for hexagonal (space group No. 189) HoNiSn alloy obtained for scalar- and full-relativistic methods are plotted in Figs. 5 and 6, respectively. In the case of hexagonal structure we have two different types of nickel atoms and hence in Figs. 5 and 6 we observe two contributions from $\mathrm{Ni}$ (2c position) and $\mathrm{Ni1}$ (1b position). The peaks are located in the similar region of energy, however the shapes are different. We observed the small change of positions and shapes of the total densities of states calculated for the hexagonal structure, however the densities of states at the Fermi energy are greater than in the case of orthorhombic structure: $N\left(E_{\mathrm{F}}\right)$ is 356.40 and 273.62 (states/(eV f.u.)) for scalar-relativistic and full-relativistic calculations, respectively.

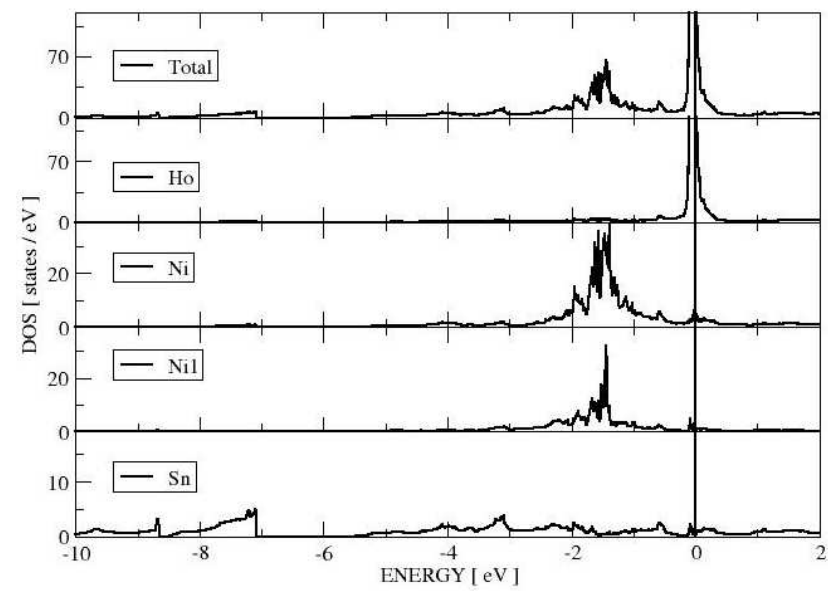

Fig. 5. Total and partial electronic density of states of HoNiSnV (scalar-relativistic, space group No. 189).

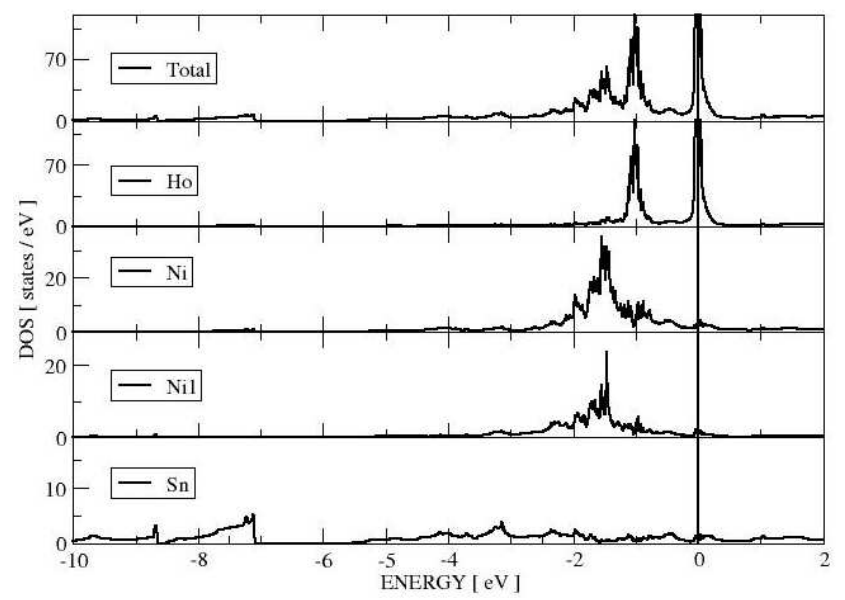

Fig. 6. Total and partial electronic density of states of HoNiSn (full-relativistic, space group No. 189).

The scalar-relativistic band calculations were performed also for orthorhombic $\mathrm{HoNiSnH}_{0.66}$ alloy. In this case a part of $4 \mathrm{~h}$ sites were occupied by hydrogen atoms. We have calculated the electronic density of states using the CPA.

The total and partial densities of states are presented in Fig. 7. The total density of states consists of a main sharp peak located at the Fermi level and the rest of the peaks are more broadened. We observed the change of position of the density of states of nickel atom located at 1b position towards the Fermi level. This effect leads to increases of the density of states at the Fermi level and $N\left(E_{\mathrm{F}}\right)=383.64$ (states/(eV f.u.)).

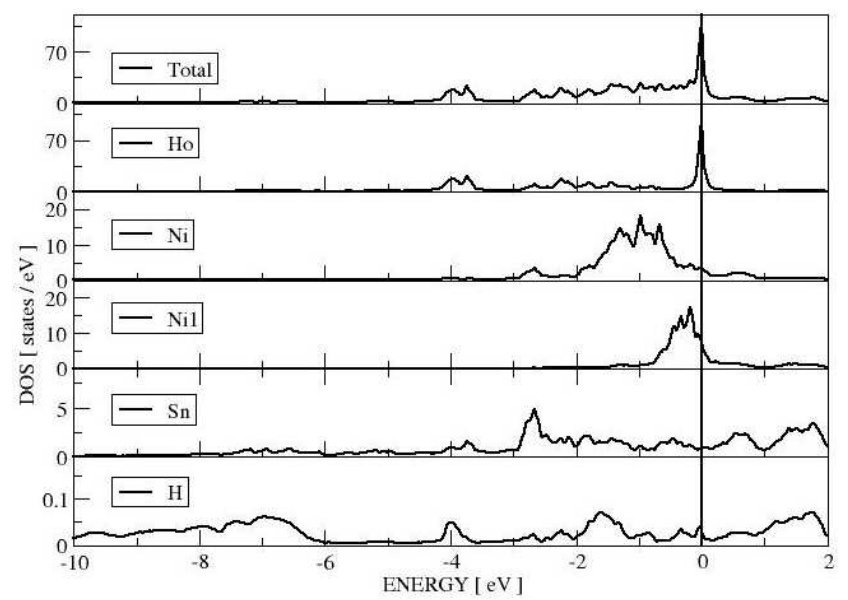

Fig. 7. Total and partial electronic density of states of $\mathrm{HoNiSnH}_{0.66}$ (scalar-relativistic, space group No. 189).

We have performed the spin-polarized band calculations for HoNiSn in the orthorhombic and hexagonal structures. The results of calculations are listed in Tables IV and V, respectively. The calculated total (spin and orbital) moment of holmium is $8.93 \mu_{\mathrm{B}}$ and $8.59 \mu_{\mathrm{B}}$ for orthorhombic and hexagonal structure, respectively. This values are close to the experimental ones [2].

The positions of peaks of Ho atom in the total density of states (Figs. 3 and 4) are not the same as was observed in X-ray photoemission (XPS) spectra [8]. It is well known that local spin density (LSD) approximation does not give correct position of peaks of lanthanides. We have performed initial calculations of the electronic structure of orthorhombic HoNiSn using LSD $+U$ approximation. The calculations were made for $1.0<U<7.0 \mathrm{eV}$ and $J=0.09 \mathrm{eV}$. We observed the change of position of peaks in the density of states. For $U=7.0 \mathrm{eV}$ the peaks from $4 f$ states of Ho were located at 2.5, 4.4 and $6.9 \mathrm{eV}$ below the Fermi level. 
TABLE IV

Magnetic moments in HoNiSn (space group No. 62, orthorhombic TiNiSn-type).

\begin{tabular}{c|c|c|c}
\hline \hline Magnetic moment $\mu_{\mathrm{B}}$ & $\begin{array}{c}\text { Scalar-relativistic } \\
\text { (spin moment) }\end{array}$ & $\begin{array}{c}\text { Full-relativistic } \\
\text { (spin moment) }\end{array}$ & Orbital moment \\
\hline Total [f.u.] & 14.37 & 13.94 & \\
Ho & 3.57 & 3.48 & 5.45 \\
$\mathrm{Ni}$ & 0.05 & 0.02 & -0.02 \\
$\mathrm{Sn}$ & -0.02 & -0.02 & 0.01
\end{tabular}

TABLE V

Magnetic moments in HoNiSn (space group No. 189, hexagonal ZrNiAl-type).

\begin{tabular}{c|c|c|c}
\hline \hline Magnetic moment $\mu_{\mathrm{B}}$ & $\begin{array}{c}\text { Scalar-relativistic } \\
\text { (spin moment) }\end{array}$ & $\begin{array}{c}\text { Full-relativistic } \\
\text { (spin moment) }\end{array}$ & Orbital moment \\
\hline Total [f.u.] & 11.79 & 11.31 & \\
Ho & 3.79 & 3.66 & 4.93 \\
Ni & 0.14 & 0.12 & -0.04 \\
Ni1 & 0.09 & 0.05 & -0.03 \\
Sn & 0.02 & 0.02 & 0.01
\end{tabular}

\section{Conclusions}

Ab initio band structure calculations have shown that the shape of the electronic structure of HoNiSn alloys in orthorhombic and hexagonal structure is similar, also the details near the Fermi energy are different. The hydrogenation changes the electronic structure of HoNiSn. In $\mathrm{HoNiSnH}_{0.66}$ the peak from $\mathrm{Ni}$ atom located at $1 \mathrm{~b}$ site is moved towards the Fermi level and hence the density of states at the Fermi level in $\mathrm{HoNiSnH}_{0.66}$ is higher than in the ordered HoNiSn system. The calculated total magnetic moment of holmium is close to the experimental value.

\section{Acknowledgments}

The work was supported by Polish State Committee for Scientific Research under the contract No. PBZ-KBN0117/T08/07.

Part of the research was done in the frame of the National Network "Strong correlated materials: preparation, fundamental research and applications".

\section{References}

[1] V.A. Yartys, O. Isnard, A.B. Riabov, L.G. Akselrud, J. Alloys Comp. 356, 109 (2003).

[2] A. Szytula, O. Isnard, V.A. Yartys, A.B. Riabov, J. Alloys Comp. 404-406, 200 (2005).

[3] J.K. Yakinthos, Ch.D. Routsi, E. Roudaut, J. Magn. Magn. Mater. 136, 65 (1994).

[4] Ch. Routsi, J.K. Yakinthos, Phys. Scr. 50, 222 (1994).

[5] K. Koepernik, H. Eschrig, Phys. Rev. B 59, 1743 (1999).

[6] K. Koepernik, B. Velicky, R. Hayn, H. Eschrig, Phys. Rev. B 55, 5717 (1997).

[7] J.P. Perdew, Y. Wang, Phys. Rev. B 45, 13244 (1992).

[8] E. Talik, T. Mydlarz, J. Kusz, H. Bohm, J. Alloys Comp. 336, 29 (2002). 